\title{
Omittable Planes
}

\author{
Branko Grünbaum * Jonathan Lenchner ${ }^{\dagger}$ \\ Submitted: Jul 16, 2010; Accepted: Aug 26, 2011; Published: Sep 2, 2011 \\ Mathematics Subject Classification: 52C35
}

\begin{abstract}
In analogy to omittable lines in the plane, we initiate the study of omittable planes in 3 -space. Given a collection of $n$ planes in real projective 3 -space, a plane $\Pi$ is said to be omittable if $\Pi$ is free of ordinary lines of intersection - in other words, if all the lines of intersection of $\Pi$ with other planes from the collection come at the intersection of three or more planes. We provide two infinite families of planes yielding omittable planes in either a pencil or near-pencil, together with examples having between three and seven omittable planes, examples that we call "sporadic," which do not fit into either of the two infinite families.
\end{abstract}

\section{Introduction}

A finite family $\mathcal{L}$ of (straight) lines in the plane determines several different objects. One of these is the aggregate associated with $\mathcal{L}$, defined as the family of points $\mathcal{P}$ in which two or more of the lines intersect, together with the lines $\mathcal{L}$ themselves. To avoid trivialities and exceptions, it is customary to stipulate that $\mathcal{P}$ consists of more than a single point. A point of $\mathcal{P}$ that is on precisely two lines is called ordinary. A line $L$ of $\mathcal{L}$ that is incident with no ordinary point is called omittable, since the deletion of $L$ from $\mathcal{L}$ does not change $\mathcal{P}$.

Figure 1 shows an example of a family $\mathcal{L}$ of 15 lines; for this $\mathcal{L}$ each of the 6 heavily drawn lines is omittable. In fact, all 4 of these lines that pass through the center are simultaneously omittable.

A famous problem, still unsolved after many decades, is to determine the best lower bound for the number $s(n)$ of ordinary points in aggregates of $n$ lines. The conjecture is that $s(n) \geq\lfloor n / 2\rfloor$ for all $n$, with a higher lower bound in case $n-3$ is a multiple of 4 . The best available result is $s(n) \geq 6 n / 13$; see Csima and Sawyer[2]. There are many accounts of the history and variants of this problem; the most recent one is that of Pretorius and Swanepoel [5].

*Dept. of Mathematics, Univ. of Washington, Seattle, WA 98195, grunbaum@math. washington.edu.

${ }^{\dagger}$ IBM T.J. Watson Research Center, 19 Skyline Drive, Hawthorne, NY 10532, lenchner@us.ibm.com. 


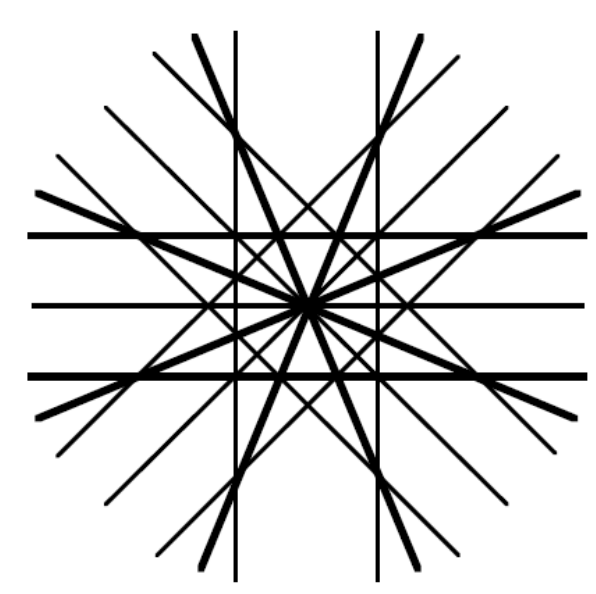

Figure 1: An aggregate of 15 lines, with 6 omittable lines shown with thickened lines.

Omittable lines have received much less attention. A recent survey of known results, open problems and conjectures is [1].

The present paper is meant to discuss another variant of this topic: omittable planes in aggregates of planes in 3-dimensional space. Although the definitions are straightforward generalizations of the ones concerning aggregates of lines in the plane, it is important to make our meaning clear. By an aggregate of planes we mean a family of planes in 3dimensional real projective space, not all of which have a point in common, together with all the lines and points that are determined by their intersections. An ordinary line of an aggregate is a line that is contained in precisely two of the planes. A plane of an aggregate is omittable if it contains no ordinary line. In other words, our concepts in 3-space arise by increasing by one the dimensions of the analogous concepts in the plane. The reason for declaring that in an aggregate of planes not all planes have a point in common is to distinguish the associated problems from their two-dimensional counterparts. If we have a family of planes with a point in common and send this point to infinity via a suitable projective transformation (in other words, a projective transformation taking any plane containing the common point, which is not a plane of the aggregate and does not contain a line of the aggregate, to the plane at infinity), we are left with planes all orthogonal to a fixed additional plane, and considerations of such a family would not differ from considerations of the aggregate of lines obtained by intersecting the planes with a fixed additional orthogonal plane.

Although the title of this paper is Omittable Planes, and in the initial phase of our investigation we worked exclusively with planes, as we delved more deeply into the subject we found that the dual setting where one considers omittable points in 3-dimensional space also provides a number of insights. Given a collection of points, $\mathcal{P}$, in 3 -space, a point $p \in \mathcal{P}$ is said to be omittable if every connecting line determined by $p$ and the other elements of $\mathcal{P}$ contains at least three points of $\mathcal{P}$. In other words, by deleting $p$ we do not delete any connecting lines. The condition that not all planes contain a common point, in the dual setting, translates to the condition that not all points lie on a common plane. 
In the discussion that follows we start by focusing almost exclusively on the problem of omittable planes, but as the discussion progresses, we pass more and more often over to the dual setting of omittable points.

Switching our attention now back to planes, the obvious questions one may ask are whether there are any omittable planes, and if so, how many and how widespread they are among various aggregates. Only few aggregates of planes in 3-space have been described in the literature. Most of these are simplicial arrangements, that is, all connected components of the complement of the planes are simplices. Besides two infinite families and 15 sporadic examples described in [3] and (in more detail) in [4], four additional examples are described in several publication of G. L. Alexanderson and J. E. Wetzel (see [6] for details and references). A perusal of all these simplicial arrangements shows that (considered as aggregates) there is not a single omittable plane in any of them. This negative result is somewhat tempered by the constructions detailed in the next section. Note that in all the examples and results we interpret the 3-dimensional real projective space as modeled by the 3-dimensional Euclidean space augmented by a "plane at infinity" and its lines and points, which we call "at infinity."

\section{Systematic constructions}

Theorem 1 The plane $\Pi$ of any aggregate of lines $\mathcal{L}$ in $\Pi$ is an omittable plane for some aggregate of planes $\mathcal{A}$.

Proof. Given an aggregate of lines $\mathcal{L}$ in the plane $\Pi$. Consider the aggregate $\mathcal{A}$ formed by $\Pi$, and, for each line $L$ of $\mathcal{L}$, two or more other planes that pass through $L$. Then clearly $\Pi$ is an omittable plane of $\mathcal{A}$.

This construction is easily seen to completely characterize the family of aggregates having at least one omittable plane. A simple illustration of this construction starts with a regular polygon $C$ in the plane $\Pi$, and constructs a bipyramid $B$ with equatorial circuit $C$. Then $\Pi$ and the planes of the faces of $B$ form an aggregate for which $\Pi$ is an omittable plane.

Theorem 1 guarantees the existence of one omittable plane in some aggregates - but are there examples of aggregates with more than one omittable plane? The positive answer again allows considerable freedom of choice. We start with a "parallels construction:"

Given two parallel planes $P$ and $Q$, we select in each plane a family of at least two parallel lines. All these lines must be parallel, but are otherwise arbitrary. Next we construct transversal planes intersecting each of $P$ and $Q$ along the lines chosen, perfectly arbitrary except that each line should be in at least two of the crossing planes. Adding the plane at infinity, and/or one or more planes parallel to $P$ and $Q$, and the construction is complete modulo one detail. We still do not have an aggregate since all the planes in question have a common point "at infinity." However, the construction can be rescued by simply repeating it, this time with a family of parallel lines in $P$ and $Q$ that are not parallel to the first family. 
Theorem 2 Any aggregate with two omittable planes is projectively equivalent to an aggregate obtained by the "parallels construction" with two or more families of parallels in different directions.

Proof. Considering the intersection of the two planes $P$ and $Q$ we see that at least one additional plane must contain that line. Using an appropriate projective transformation we map the additional plane to infinity, thus making the two omittable planes parallel. Now starting with any of the families of parallel lines in the planes $P$ and $Q$, we see that there must be at least two families, and the "parallels construction" applies.

It is worth observing that if the aggregate includes at least two planes parallel to $P$ and $Q$, then $P$ and $Q$ are simultaneously omittable.

In analogy to the notion of a "pencil of lines" we say that a collection of planes forms a "pencil" (i.e. a "pencil of planes") if the planes all intersect in a common line.

Theorem 3 Any pencil of planes $\mathcal{P}$ can be amongst the set of omittable planes for some aggregate. Therefore there is no limit to the number of omittable planes in an aggregate. Moreover, for any given pencil of planes $\mathcal{P}$ the size of an aggregate containing $\mathcal{P}$ amongst its omittable planes can be arbitrarily large.

Proof. Without loss of generality we may take the line of intersection of the pencil of planes to be at infinity, so it is sufficient to prove the claimed results for the case where the pencil is an arbitrary collection of mutually parallel planes. The case where there are just two planes in the pencil is covered by Theorem 2, so we may assume that the pencil has at least three planes.

We start with an arbitrary collection of lines parallel to the $y$-axis in the $z=0$ plane. By Theorem 2 of [1], there is a line aggregate in the $z=0$ plane having these lines as its set of omittable lines. Form a collection of planes by passing planes through each line in the aggregate, orthogonal to the $z=0$ plane. This collection of planes is not yet an aggregate since the planes all have the point at infinity in the direction of the $\mathrm{y}$-axis in common. But now imagine the entire assembly of planes as a rigid body and rotate all planes about the $x$-axis by some small angle $\theta=2 \pi \epsilon$, for $\epsilon$ irrational. The vertical planes will stay fixed, and the other planes will all move. For our aggregate, we take the collection of planes in the original assembly plus the new, rotated planes. Note that the vertical planes are all still omittable, since any line intersection formed on such a plane is either a line intersection formed with planes from the unrotated or rotated aggregate, and in either case it cannot be ordinary. Since $\epsilon$ was chosen to be irrational, we can do this rotation as many times as we wish, yielding aggregates of arbitrarily large size having the starting set of parallel planes as omittable planes.

At a minimum, all but two of such an omittable pencil of planes are simultaneously omittable.

Theorem 4 For any $g \geq 3$ there is an aggregate of planes $\mathcal{L}$ having a set of omittable planes of size $g$ all forming a pencil and $n=|\mathcal{L}|=5 \mathrm{~g}$. 
Proof. By Theorem 1 in [1], for any $g \geq 3$ there is an aggregate of lines $\mathcal{L}$ in $2 D$ with $g$ omittable lines forming a pencil and $|\mathcal{L}|=3 g$. Using this line aggregate as the starting point in the proof of Theorem 3, with just a single rotation of the original all-vertical planes, yields an aggregate of size $5 g$ with $g$ omittable planes.

Theorem 5 For any $g \geq 2$, an aggregate of planes $\mathcal{L}$ having a set of $g$ omittable planes forming a pencil must be of size $n=|\mathcal{L}| \geq 5 \mathrm{~g}$.

Proof. Dualize the planes to points. Since the omittable planes all formed a pencil, the now omittable points all lie on a single line - call this line $\ell$. There must be some point $r \in \mathcal{P}$ not on $\ell$. Consider the plane spanned by $r$ and $\ell$. For simplicity of exposition and without loss of generality take $\ell$ to be the line at infinity in the $z=0$ plane and $r$ to be a finite point in that plane. Consider the convex hull, $C$, of the points of $\mathcal{P}$ contained in the finite part of the $z=0$ plane. Since $r$ lies somewhere inside $C$, possibly on $C, C$ contains at least one point. The line $\ell$ contains at least two points, call two of them $p_{1}$ and $p_{2}$. Each point is omittable, hence there is an additional point on the line determined by $p_{1}$ and $r$ in addition to $p_{1}$ and $r$, as well as an additional point on the line determined by both $p_{2}$ and $r$ in addition to $p_{2}$ and $r$. Since both of these lines lie in the $z=0$ plane the additional points lie in this plane. Moreover, the additional points are finite and distinct. Call these points $r^{\prime}$ and $r^{\prime \prime}$. The three points $r, r^{\prime}$ and $r^{\prime \prime}$ are not collinear. The convex hull, $C$, of all the finite points in the plane is therefore 2-dimensional. Every point in the omittable pencil of points determines two support parallel lines of $C$ : consider lines in the direction of each $p \in \ell$ lying in the $z=0$ plane coming in respectively from positive and negative infinity - since $C$ is 2-dimensional they will intersect $C$ once on one side and once on the other side. Since each point in the pencil of points is omittable, each of the support lines contains at least two vertices of $C$. By convexity, each vertex of $C$ is associated with at most two omittable points (i.e. lies on at most two supporting lines). Therefore $C$ has at least $2 g$ vertices. Since not all points in the aggregate lie in one plane (equivalently, in the primal, not all planes have a common point) there is another point $q$ not in the plane containing all the points thus far accounted for. Applying the same argument as before to the plane spanned by $q$ and $\ell$ we obtain another $2 g$ points contained in this plane but not contained in $\ell$ - and hence too not in the first plane. We thus have at least $5 g$ points in total and the theorem is proved.

Theorem 6 For any $g=2 k \geq 4$ ( $k$ an integer) one can find an aggregate with $g$ omittable planes forming a near pencil and either $n=7 g-6$ or $n=7 g-4$ total planes.

Proof. We perform this construction in the dual where we will place points on the three parallel planes $z=0, z=1$ and $z=-1$. On the $z=0$ plane we place points at the vertices of a regular $2(g-1)$-gon and another point at its center. In the $z= \pm 1$ planes we place an exact copy of the vertex points (in other words a copy of all vertex points translated by $(0,0, \pm 1))$. To this aggregate we add the $g-1$ points at infinity corresponding to the directions determined by the center and the vertices of the regular $2(g-1)$-gon in any one of the planes. In Figure 2 we illustrate this construction in the 


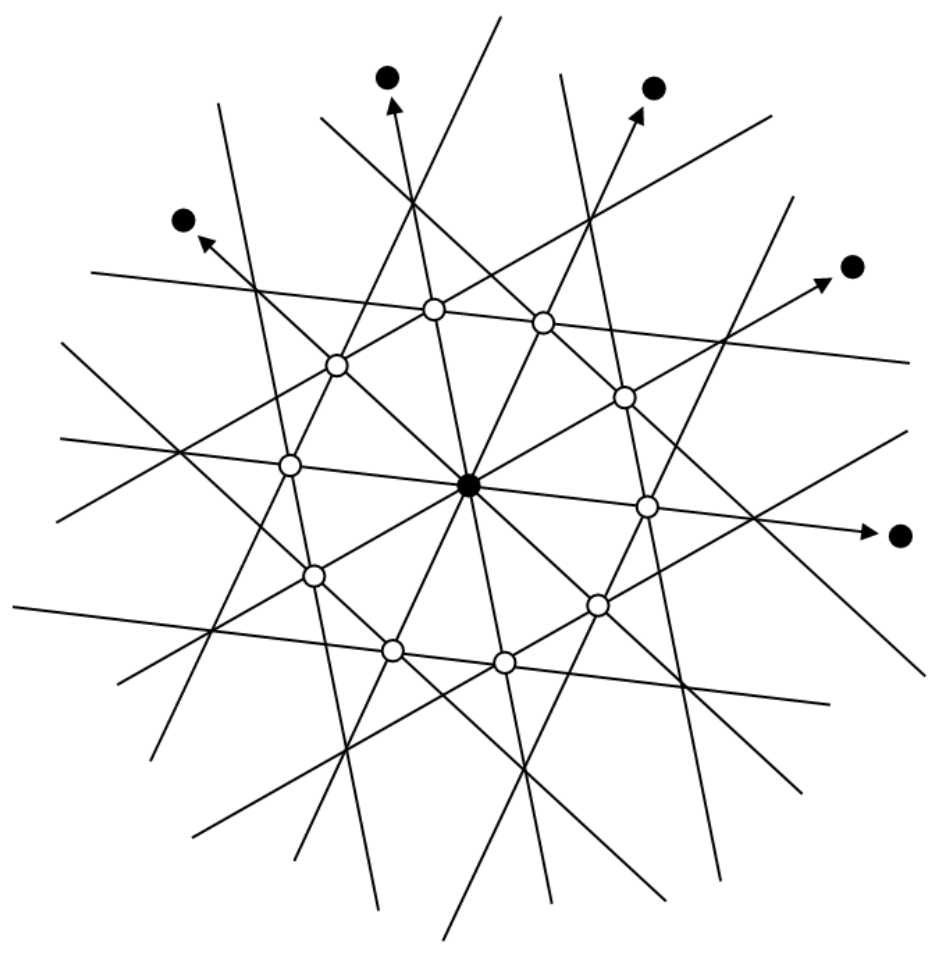

Figure 2: Placement of points in the $z=0$ plane in the proof of Theorem 6 . The omittable points forming a near-pencil are shown in black.

$z=0$ plane for $g=6$, so the polygon is a regular $2(g-1)=10$-gon. The points at infinity and the center point are all omittable and the aggregate consists of $7 g-6$ total points. If we add the center points in the $z= \pm 1$ planes we get an aggregate with the same set of omittable points and $7 g-4$ total points.

\section{Sporadic examples}

By a "sporadic" aggregate we mean any aggregate having at least 3 omittable planes, and in which the omittable planes do not all form a pencil or near pencil satisfying the conditions of Theorem 6. In order to present the aggregates in question we need to overcome the well-known difficulty of illustrating such objects with a measure of intelligibility. Our attempt at such a presentation follows.

We start by describing the aggregate. In the examples this is done using an easily recognizable convex polyhedron, chosen because it has a high degree of symmetry. Next we show a diagram depicting the polyhedron, on which we indicate, using different colors, representatives of several families of planes. We describe geometrically one of the planes in each family, and stipulate that the other members of its family are planes equivalent under symmetries of the polyhedron to the one described. In some cases we describe the planes of the aggregate by their equations in a suitable system of coordinates. For easier description of each example we label all members of each family by one appropriate letter. 
As a second step we have to show that each of the planes we claim to be omittable is in fact omittable. In most cases we do this by showing the trace on the plane $P$ in question of all the other planes, and the labels just mentioned are placed near the respective lines of the trace to indicate planes of which family or families generate the particular line. The verification of the omittability of $P$ is then very simple, requiring only that one check that each line present in the trace arises from the intersection of at least two additional planes.

Example 1 There is a sporadic aggregate of 15 planes such that 5 of the planes are omittable.

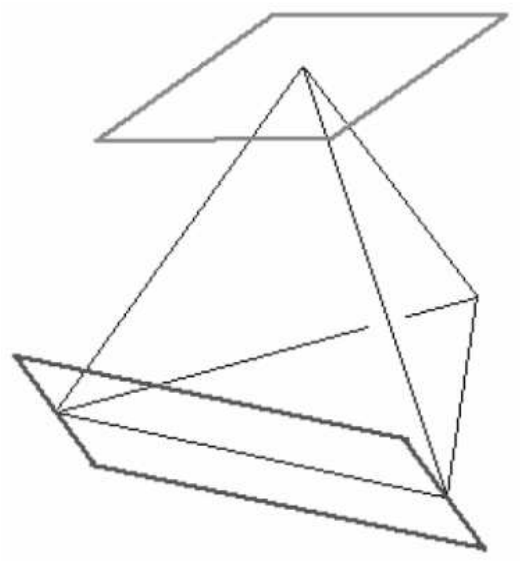

(a)

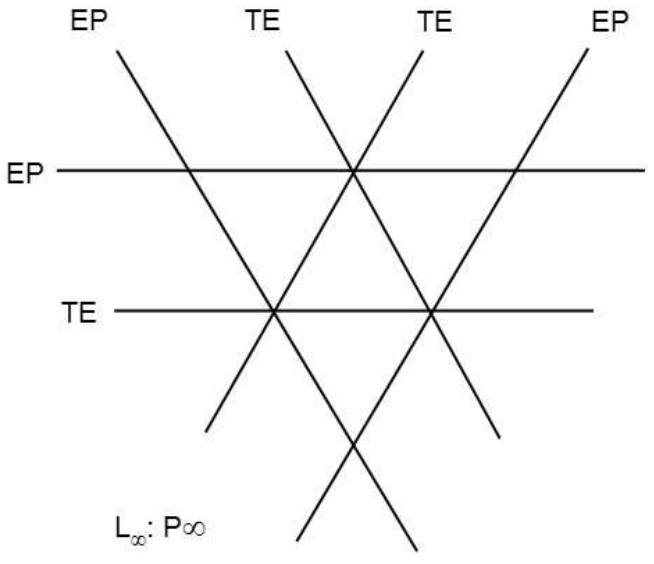

(b)

Figure 3: An aggregate of 15 planes, based on the regular tetrahedron, with 5 omittable planes. The line aggregate on the right shows the trace of the various planes on one of the $T$-planes. $L_{\infty}$ denotes the line at infinity on this $T$-plane.

We start with a (regular) tetrahedron as shown in Figure 3(a), and construct the family as follows:

$4 T$-planes, each the affine span of one of the faces of the tetrahedron.

$6 \quad E$-planes, each supporting an edge and parallel to the opposite edge.

$4 \quad P$-planes, each through a vertex and parallel to the opposite face.

$1 \infty$-plane at infinity.

Figure 3(b) shows one of the $T$-planes, with lines that are traces of the other 14 planes. Since each line is the trace of two of the other planes, the $T$-plane is omittable, as are obviously the remaining $T$-planes. This accounts for four of the omittable planes. The fifth is the plane at infinity; its omittability is obvious since the other planes come in parallel pairs: The six $E$-planes form three parallel pairs, and each $T$-plane is parallel to a $P$-plane. 
Example 2 There is another sporadic aggregate of 15 planes with 5 omittable planes.

Figure 4 shows the aggregate which is based on the 3-sided Archimedean prism. The planes of the aggregate are as follows:

$2 H$-planes, each the affine span on one of the bases of the prism.

$3 \quad F$-planes, each the affine span on one of the mantle faces (squares) of the prism.

$3 E$-planes, each supporting a vertical edge of the prism and parallel to the opposite face.

$6 \quad S$-planes, slanted planes, each containing one of the horizontal edges and the vertex opposite it in the other basis.

$1 \infty$-plane, the plane at infinity.

Figure 5 shows the traces of the other planes in one of the $F$-planes and in one of the $H$-planes. Each line is the trace of two of the remaining planes and hence the $F$-planes and the $H$-planes are omittable.

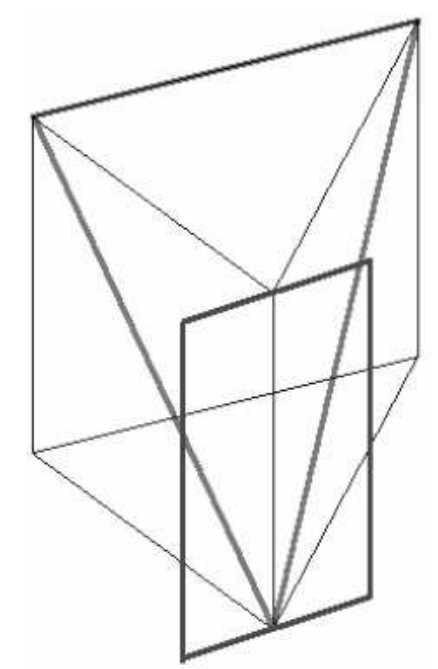

Figure 4: An aggregate of 15 planes, derived from a prism, with 5 omittable planes.

The next example has the largest number of omittable planes among the sporadic aggregates we have found.

Example 3 There exists a sporadic aggregate $\mathcal{A}$ with 33 planes and 7 omittable planes.

Before describing this example in detail, let us introduce a little bit of notation and terminology:

Definition 1 An aggregate is said to have order $(\boldsymbol{n}, \boldsymbol{g})$ if it consists of $n$ planes (points) and has exactly $g$ omittable planes (points). We sometimes refer to such an aggregate as an $(\boldsymbol{n}, \boldsymbol{g})$-aggregate. 
We start with a somewhat bigger example, in the sense that $n=|\mathcal{A}|=39$. The construction starts with the standard cube given by $|x| \leq 1,|y| \leq 1,|z| \leq 1$. The aggregate consists of the following 39 planes, see Figure 6(a).

$6 \quad C$-planes (cube planes) $\pm x=1, \pm y=1, \pm z=1$.

$6 \quad D$-planes (diagonal planes) $\pm x=y, \pm y=z, \pm z=x$.

$8 T$-planes (tetrahedral planes) $\pm x \pm y \pm z=1$.

$12 S$-planes (support planes) $\pm x \pm y=2, \quad \pm y \pm z=2, \quad \pm z \pm x=2$.

$6 \quad P$-planes (parallel planes) $\pm x=3, \pm y=3, \pm z=3$.

$1 \infty$-plane at infinity.

We claim that each of the six $C$-planes is omittable from $\mathcal{A}$. Indeed, due to symmetry, we need only check that the $C$-plane $z=1$ is omittable. But this is obvious from the attached diagram, since each line in that plane belongs to two or three other planes. The seventh omittable plane is the plane at infinity - its omittability follows at once upon observing that all the other planes come in parallel pairs (or larger parallel sets).

Note that all the $C$-planes and the plane at infinity can be simultaneously omitted. As a result, by successively deleting any of these planes we obtain aggregates of orders $(38,6),(37,5),(36,4)$ and $(35,3)$.

Moreover, any subset of the $D$-planes, or all of them can be dropped from the original aggregate $\mathcal{A}$, thus giving aggregates with between 33 and 38 planes, and seven omittable planes.

In Figure 6 we show the traces in the $C$-plane $z=1$ of the 38 other planes in the original construction. Since each of the traces arises from two or more planes even disregarding the $D$-planes, the validity of our claims regarding Example 3 follows.

In the next section we show that there are precisely 7 omittable planes in this 39 plane aggregate as well as in the 33 plane aggregate (and all those in between).

The next example is less interesting for its own sake, but possibly it is pointing a way to the construction of larger aggregates.

Example 4 There is a sporadic aggregate of 18 planes with 3 omittable planes

The construction of such an aggregate is illustrated in Figure 8. There are:

$2 H$-planes, each the affine span of one of the horizontal bases of the prism.

$3 \quad F$-planes, each the affine span of one of the mantle faces of the prism.

$3 \quad M$-planes of mirror symmetry in vertical mirrors.

6 S-planes, slanted planes, each containing one horizontal edge and a vertex of the opposite basis.

3 E-planes, each parallel to a vertical edge and bisecting four horizontal edges.

$1 \infty$-plane at infinity.

As is easily verified, each $F$-plane is omittable since the trace of each of the other planes on an $F$-plane is shared by one or two other planes. 


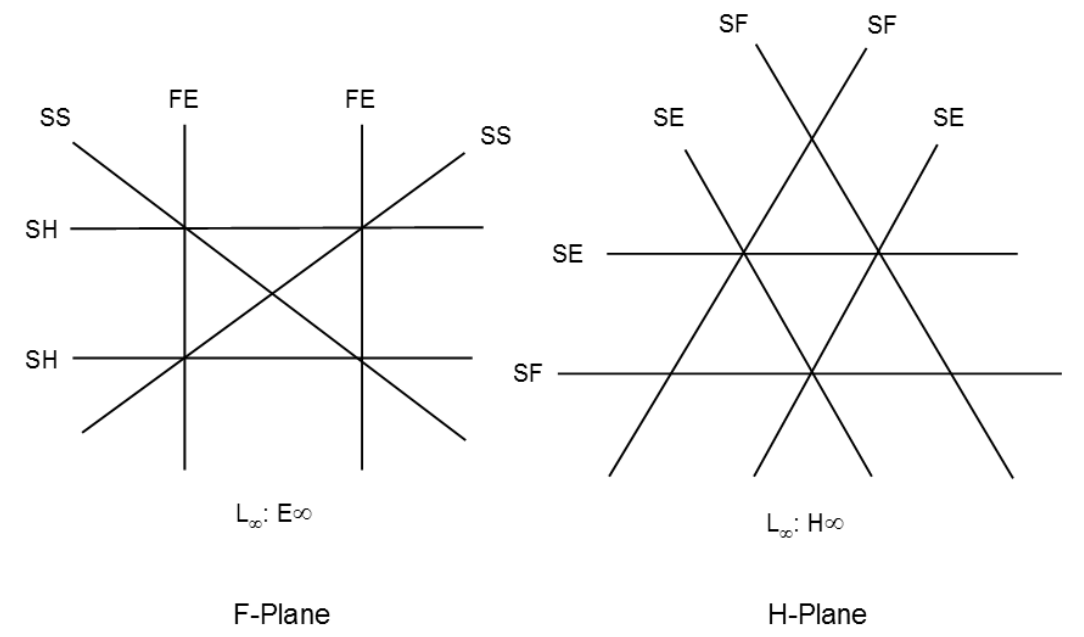

Figure 5: Traces on the 5 omittable planes - the three $F$-planes and two $H$-planes.
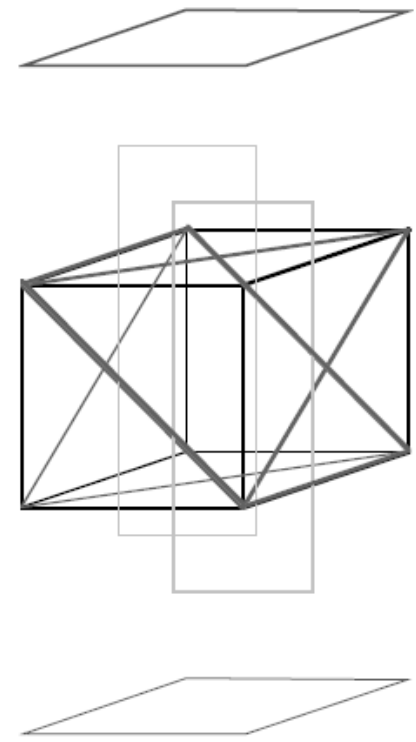

Figure 6: A representation of the aggregate of 39 planes used in Example 3. The aggregate includes the plane at infinity. 


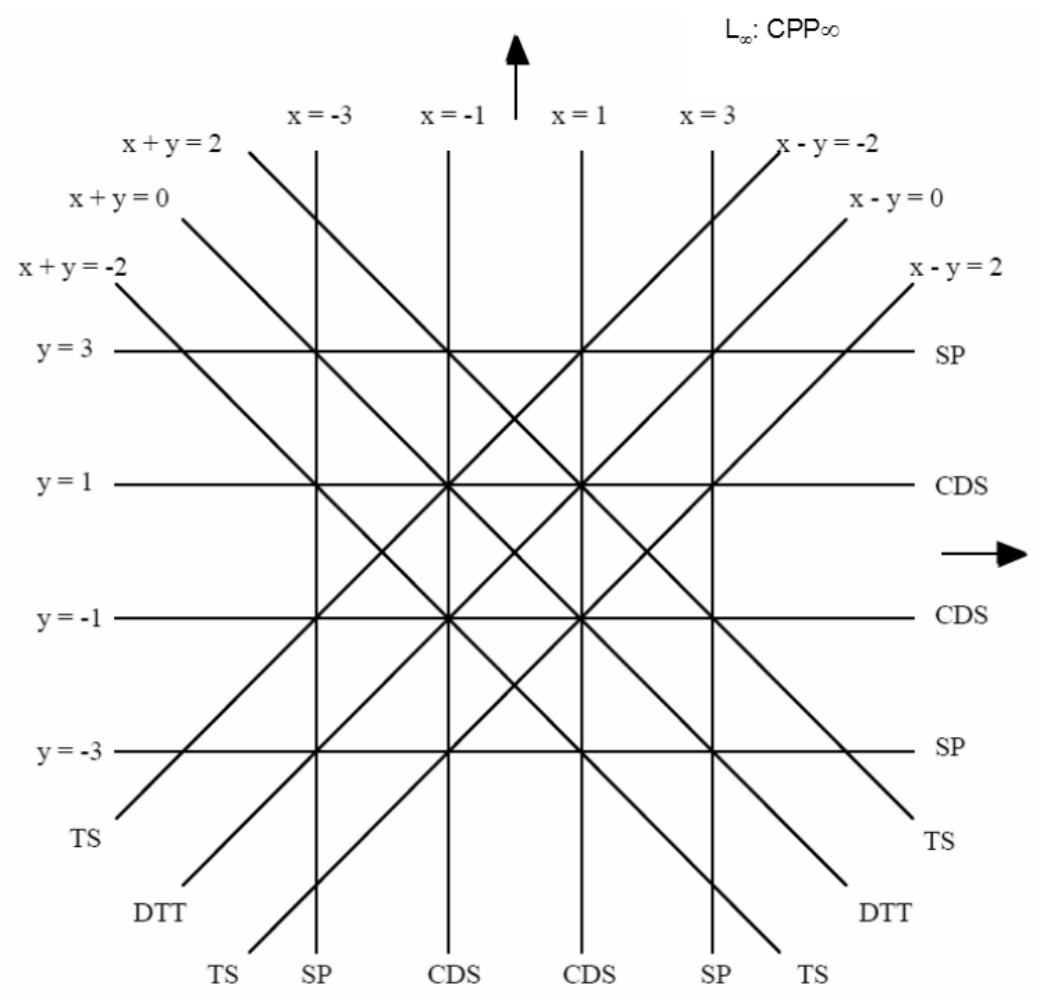

Figure 7: Traces of the other planes on the $C$-plane $z=1$.
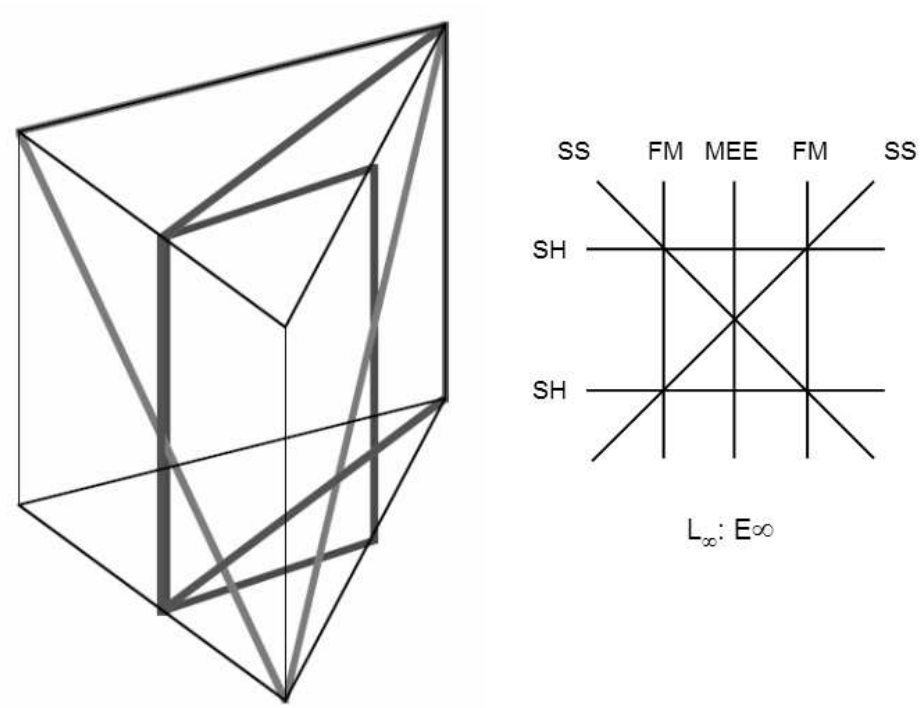

Figure 8: The 18 planes (including the plane at infinity) that form an aggregate with 3 omittable planes; each $F$-plane is omittable, as shown by the traces of its intersections with the other planes. 


\section{Towards a More Systematic Understanding of the Sporadic Aggregates}

After discovering the above-mentioned sporadic aggregates many questions remained. Was it possible to find arbitrarily large sporadic aggregates, either in terms of the number of omittable planes, or even the total number of planes? Was it possible to find a sporadic aggregate of 15 or fewer planes and just 3 or 4 omittable planes? Were the two $(15,5)$ aggregates of Examples 1 and 2 really distinct?

We were able to answer several of these questions. First let us turn our attention to the question of whether the two $(15,5)$ aggregates of Examples 1 and 2 are really distinct. It at first seemed obvious to us that these two aggregates must be distinct. One of the reasons for this opinion was that the omittable planes in Example 1 form two transitivity classes under symmetries, with one class consisting of four planes, the other consisting of a single plane. In contrast, the five omittable planes in Example 2 form transitivity classes of 2 and of 3 planes. The difficulty of intelligibly depicting complete aggregates of planes seemed the main obstacle to proving that the two examples were not isomorphic. However, many attempts to find combinatorial differences between the two aggregates failed, eventually leading us to try to reach a contradiction from the assumption that they are isomorphic.

We need to be precise, however, by what we mean when we say that two aggregates are isomorphic.

Definition 2 Let $\mathcal{A}$ and $\mathcal{B}$ be two aggregates of planes. Then $\phi: \mathcal{A} \rightarrow \mathcal{B}$ is an isomorphism of aggregates iff $\phi$ is a bijection of planes and the induced mapping of lines and points of intersection are bijections as well. In other words, for any planes $\Pi_{1}, \ldots, \Pi_{k}$ in $\mathcal{A}, \Pi_{1} \cap \ldots \cap \Pi_{k}$ is a line in $\mathcal{A}$ iff $\phi\left(\Pi_{1} \cap \ldots \cap \Pi_{k}\right)=\phi\left(\Pi_{1}\right) \cap \ldots \cap \phi\left(\Pi_{k}\right)$ is a line in $\mathcal{B}$, and, moreover, $\Pi_{1} \cap \ldots \cap \Pi_{k}$ is a point in $\mathcal{A}$ iff $\phi\left(\Pi_{1} \cap \ldots \cap \Pi_{k}\right)=\phi\left(\Pi_{1}\right) \cap \ldots \cap \phi\left(\Pi_{k}\right)$ is a point in $\mathcal{B}$.

Note that if $\mathcal{A}$ and $\mathcal{B}$ are isomorphic as aggregates they need not be isomorphic as arrangements - in other words an aggregate isomorphism need not preserve facial structure. Indeed any two arrangements with the same number of planes such that no three planes intersect in a common line and no four planes intersect in a common point are isomorphic as aggregates (via an arbitrary bijection of planes), but not necessarily as arrangements.

In fact, the aggregates from Examples 1 and 2 turn out to be isomorphic. Clearly any isomorphism must take the omittable planes in one aggregate to the omittable planes in the other, and it is then easy to guess a possible mapping between the aggregates that proves despite appearances and expectations that they are isomorphic. In Figure 9 we show a labeling and correspondence of all the planes of each of the two aggregates and a correspondence of these labels that gives rise to this isomorphism. 


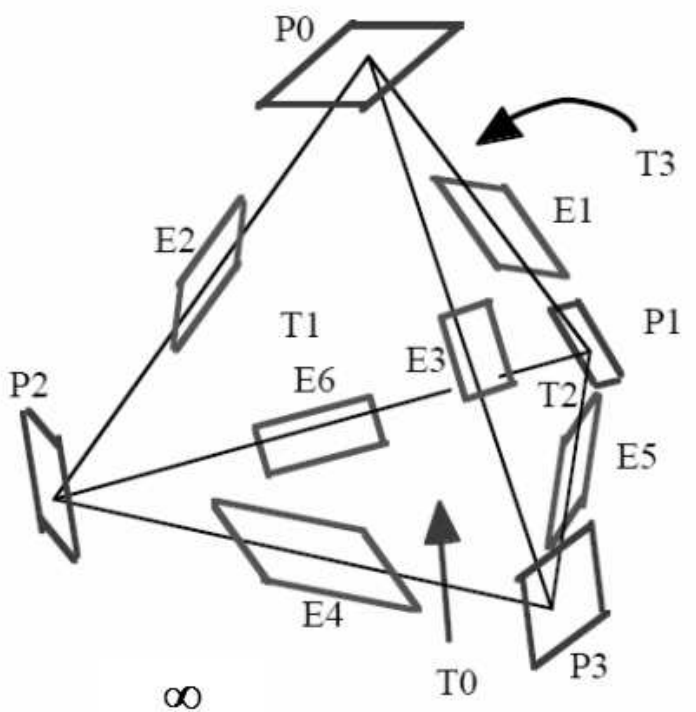

4 T-planes (spans of the faces of the tetrahedron) black

6 E-planes (planes supporting an edge, parallel to opposite edge) red

4 P-planes (planes through a vertex, parallel to opposite face) blue

$1 \infty$ - plane at infinity

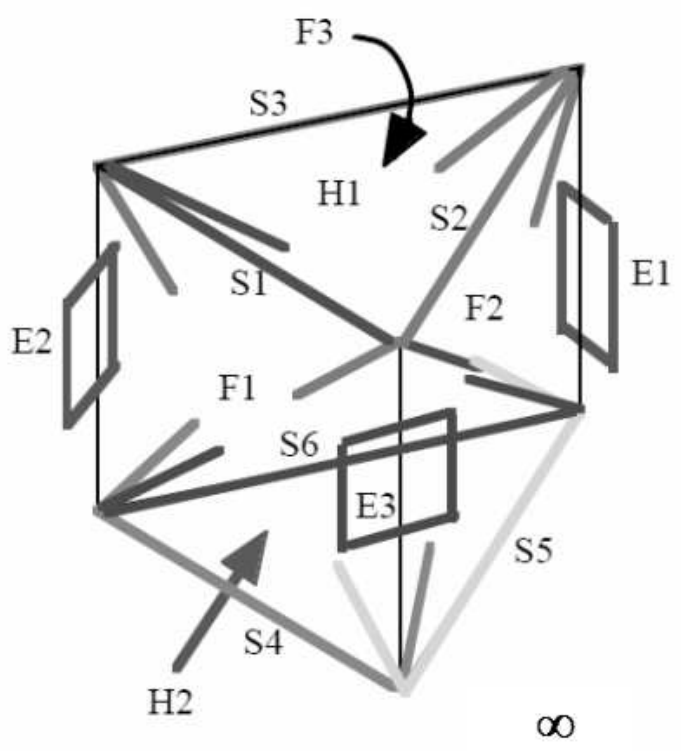

2 H-planes (spans of horizontal faces) black

3 F-planes (spans of vertical faces) black

6 S-planes (slanted planes) green and yellow hues

3 F-nlanes (edge-support planes) blue

$1 \infty$ - plane at infinity

Figure 9: The fully labeled aggregates of Examples 1 and 2, used to show that they are isomorphic.

In tabular form, this correspondence (which for later reference we shall call $\Phi$ ) is as follows:

\begin{tabular}{|l|c|c|c|c|c|c|c|c|c|c|c|c|c|c|c|}
\hline Tetrahedron & T0 & $\infty$ & P0 & T1 & T2 & T3 & E1 & E2 & E3 & E4 & E5 & E6 & P1 & P2 & P3 \\
\hline Prism & H1 & H2 & $\infty$ & F1 & F2 & F3 & E1 & E2 & E3 & S1 & S2 & S3 & S4 & S5 & S6 \\
\hline
\end{tabular}

The detailed computation that $\Phi$ induces an aggregate isomorphism is quite tedious. We present such a verification only for the traces of planar intersections on the T1-plane of the tetrahedral example and the corresponding traces on $\Phi(T 1)$ in the prismatic example. Doing similarly for the remaining $T$ planes as well as the $E, P$ and $\infty$ planes would complete the verification. The detailed traces on the $T 1$ and $\Phi(T 1)$ planes are given in Figure 10 and the computation follows. When verifying a line of intersection we check that the mapping $\Phi$ takes a line of intersection in the plane $T 1$ to a line of intersection in the plane $F 1=\Phi(T 1)$. All lines of intersection in $T 1$ and $F 1$ come at the intersection of the same number of planes (3) so there is nothing to check there. When verifying a point of intersection we check that $\Phi$ takes a point of intersection on $T 1$ to a point of intersection on $F 1$ and, moreover, that each such point comes at the intersection of the same number 
of planes. The check marks indicate that this verification has been performed for each of the intersections.

Lines of intersection:

$\Phi(E 6 \cap P 2)=\Phi(E 6) \cap \Phi(P 2)=S 3 \cap S 5$, so $E 6 P 2 \mapsto S 3 S 5 \checkmark$
$\Phi(T 2 \cap E 3)=\Phi(T 2) \cap \Phi(E 3)=F 2 \cap E 3$, so $T 2 E 3 \mapsto F 2 E 3 \checkmark$
$\Phi(T 3 \cap E 2)=\Phi(T 3) \cap \Phi(E 2)=F 3 \cap E 2$, so $T 3 E 2 \mapsto F 3 E 2 \checkmark$
$\Phi(E 5 \cap P 3)=\Phi(E 5) \cap \Phi(P 3)=S 2 \cap S 6$, so $E 5 P 3 \mapsto S 2 S 6 \checkmark$
$\Phi(E 1 \cap P 0)=\Phi(E 1) \cap \Phi(P 0)=E 1 \cap \infty$, so $E 1 P 0 \mapsto E 1 \infty \checkmark$
$\Phi(T 0 \cap E 4)=\Phi(T 0) \cap \Phi(E 4)=H 1 \cap S 1$, so $T 0 E 4 \mapsto H 1 S 1 \checkmark$
$\Phi(P 1 \cap \infty)=\Phi(P 1) \cap \Phi(\infty)=S 4 \cap H 2$, so $P 1 \infty \mapsto S 4 H 2 \checkmark$

Points of intersection:

$\Phi(E 6 P 2 \cap E 1 P 0)=\Phi(E 6 P 2) \cap \Phi(E 1 P 0)=S 3 S 5 \cap E 1 \infty \checkmark$

$\Phi(T 2 E 3 \cap T 3 E 2 \cap E 1 P 0)=\Phi(T 2 E 3) \cap \Phi(T 3 E 2) \cap \Phi(E 1 P 0)=F 2 E 3 \cap F 3 E 2 \cap E 1 \infty \checkmark$ $\Phi(E 5 P 3 \cap E 1 P 0)=\Phi(E 5 P 3) \cap \Phi(E 1 P 0)=S 2 S 6 \cap E 1 \infty \checkmark$

$\Phi(E 6 P 2 \cap T 0 E 4 \cap T 3 E 2)=\Phi(E 6 P 2) \cap \Phi(T 0 E 4) \cap \Phi(T 3 E 2)=S 3 S 5 \cap H 1 S 1 \cap F 3 E 2 \checkmark$

$\Phi(T 2 E 3 \cap E 5 P 3 \cap T 0 E 4)=\Phi(T 2 E 3) \cap \Phi(E 5 P 3) \cap \Phi(T 0 E 4)=F 2 E 3 \cap S 2 S 6 \cap H 1 S 1 \checkmark$

$\Phi(E 6 P 2 \cap E 5 P 3)=\Phi(E 6 P 2) \cap \Phi(E 5 P 3)=S 3 S 5 \cap S 2 S 6 \checkmark$

$\Phi(E 6 P 2 \cap T 2 E 3 \cap P 1 \infty)=\Phi(E 6 P 2) \cap \Phi(T 2 P 3) \cap \Phi(P 1 \infty)=S 3 S 5 \cap F 2 E 3 \cap S 4 H 2 \checkmark$

$\Phi(T 3 E 2 \cap E 5 P 3 \cap P 1 \infty)=\Phi(T 3 E 2) \cap \Phi(E 5 P 3) \cap \Phi(P 1 \infty)=F 3 E 2 \cap S 2 S 6 \cap S 4 H 2 \checkmark$

$\Phi(E 1 P 0 \cap T 0 E 4 \cap P 1 \infty)=\Phi(E 1 P 0) \cap \Phi(T 0 E 4) \cap \Phi(P 1 \infty)=E 1 \infty \cap H 1 S 1 \cap S 4 H 2 \checkmark$

In light of the existence of a $(15,5)$ aggregate, the following result was again somewhat surprising:

Theorem 7 Any aggregate of planes having at least 3 omittable planes must consist of at least 15 planes in total.

Proof. Dualize the planes to points. If the 3 omittable points are collinear then by Theorem 5 there must be at least $5 g=15$ total points. Hence assume the omittable points are not collinear. Call the omittable points $x, y$ and $z$ and without loss of generality assume that the affine span of $x, y, z$ is the plane at infinity. Since an aggregate of planes cannot all intersect at a common point, in the dual not all points can be coplanar, so there must be at least one finite point in addition to $x, y$ and $z$. Call this point $q$. Just considering two of the omittable points, say $x$ and $y$, in the plane spanned by $x, y$ and $q$, there must be at least 3 additional finite points (by the argument from Theorem 5 with $g=2$ ). Label these four finite points, coplanar with $x$ and $y: q, r, s$ and $t$. Now consider the line through $z$ and, say, $q$. Since $z$ is omittable, the line must contain another point, different from any we have listed so far, since $z$ is not coplanar with all the other coplanar points. Moreover, since $z$ is infinite and $q$ is finite, the additional point must be finite. Call the additional point $a$. Considering the plane spanned by $x, y$ and $a$, we see again that this plane must contain 3 additional finite points, again distinct from any we have labeled thus far. Call the four points coplanar with $x$ and $y$ on this new second parallel plane $a, b, c$ and $d$. Finally, let us consider the plane at infinity. The 8 points $q, r, s, t, a, b, c$, and $d$ are finite and so do not lie on this plane. However, $x, y$ and $z$ are 
omittable and so must be omittable with respect to points on this plane at infinity. It is very easy to see that any such plane must contain at least 7 total points. If we rotate the plane at infinity so that it is a finite plane, and $x, y$ are on the line at infinity and omittable, then there must be 4 finite points, and to keep $x$ and $y$ omittable on the line at infinity, an extra point on the line at infinity as well. Thus, in total we have the 8 finite points and 7 points on the plane at infinity for a total of 15 points.

In light of the above theorem, we next wondered whether it was actually possible to form a $(15,3)$ aggregate. Since we had been deriving a fair amount of insight by moving to the dual setting of omittable points rather than omittable planes we decided to investigate the duals of some of the sporadic aggregates which we have mentioned in the previous section. The duality map we used for these investigations was the incidence preserving map which takes the plane given by $\{(x, y, z): z=u x+v y-w\}$ to the point $(u, v, w)$. For equations of planes which do not contain a $z$-term the trick is to write the equation formally as $z=\infty u x+\infty v y-\infty w$ and then "divide" by $\infty$ to get zero on the left hand side. The equation $0=u x+v y-w$ then maps to the point at infinity in the $(u, v, w)$ direction which we donote by $(u, v, w)_{\infty}$. Using this map, the aggregate of Example 2 is transformed into a point aggregate as follows:

$H$-planes:

$$
\begin{aligned}
z=-1 & \mapsto(0,0,1) \\
z=0 & \mapsto(0,0,0)
\end{aligned}
$$

F-planes:

$$
\begin{aligned}
x=0 & \mapsto(1,0,0)_{\infty} \\
y=0 & \mapsto(0,1,0)_{\infty} \\
x+y=1 & \mapsto(1,1,1)_{\infty}
\end{aligned}
$$

E-planes:

$$
\begin{aligned}
x=1 & \mapsto(1,0,1)_{\infty} \\
y=1 & \mapsto(0,1,1)_{\infty} \\
x+y=0 & \mapsto(1,1,0)_{\infty}
\end{aligned}
$$

$S$-planes:

$$
\begin{aligned}
z=-x & \mapsto(-1,0,0) \\
z=-y & \mapsto(0,-1,0) \\
z=x+y-1 & \mapsto(1,1,1) \\
z=y-1 & \mapsto(0,1,1) \\
z=x-1 & \mapsto(1,0,1) \\
z=-x-y & \mapsto(-1,-1,0)
\end{aligned}
$$


$\infty$-plane:

$$
\infty \mapsto(0,0,1)_{\infty}
$$

Since all points in the point aggregate lie on either the $w=0$ plane, the $w=1$ plane, or the plane at infinity, a useful visualization of the aggregate is as a series of planar layers as depicted schematically in Figure 11. The points in the plane at infinity are projected onto the $w=1$ plane. [To see how this projection works, say for the point $p=(1,1,1)_{\infty}$, write $p$ in homogeneous coordinates in $(u, v, w, t)$-space as $p=\lambda(1,1,1,0), \lambda \in \mathbb{R}$. Then $p$ intersects the $w=1$ plane at $(1,1,1,0)$. Since all points at infinity have $t$ coordinate equal to zero, when representing the intersection with the $w=1$ plane we can drop the last two coordinates, and so, in Figure 11, $p$ becomes the point $(1,1)$.] The infinite points on this projection could just as well have been placed on either or both of the $w=0$ and $w=1$ planes, since the points lie in these planes as well. In this and all subsequently discussed aggregates we choose to display the infinite points just on the plane at infinity so that the total points depicted sums to the number of points in the aggregate. Keeping in mind that a point appearing at $(u, v)$ in the projected plane at infinity really means the point at infinity in the $(u, v, 1)$ direction - i.e. $(u, v, 1)_{\infty}$ - it is easy to verify that the points marked in red are omittable. The question of whether there is a $(15,3)$ aggregate can now be readily answered. Indeed, it is very easy to keep the omittable points in the $\infty$-plane omittable while at the same time forcing the two finite points which are now omittable to be not so - e.g. we keep the points in the finite planes fixed and move the points that make up the first quadrant-placed unit square in the projected plane at infinity so they make up an origin-centered square of length, say 2 , e.g. moving the points $\{(1,0,1),(0,1,1),(0,0,1)\}$ to $\{(-1,1,1),(-1,-1,1),(1,-1,1)\}$ while keeping $(1,1,1)$ fixed. See Figure 12 . The three previously omittable points in the plane at infinity are still omittable with respect to both the finite points (since the finite points have not moved) and also - by design - with respect to the infinite points. Moreover, it is easily checked that neither of the previously omittable finite points are now omittable and that none of the previously non-omittable finite points have become omittable. Thus a $(15,3)$ aggregate is possible.

Using this planar layers view of point aggregates we can also readily show that there are aggregates of orders $(15+3 k, 3)$ for any integer $k \geq 0$. The trick is to place additional points in the plane at infinity keeping the currently omittable points omittable. Each finite point already lies on an ordinary line with a point at infinity, and likewise each nonomittable infinite point lies on an ordinary line with some finite point, hence by placing additional points at infinity no currently non-omittable point can be made omittable. Thus, to this end, for each $k \geq 1$, place three points at $\left(\frac{1}{k+1}, \frac{1}{k+1}\right),\left(\frac{1}{k+1}, 1\right),\left(1, \frac{1}{k+1}\right)$.

Given that a $(15,3)$ aggregate is possible and that we can construct aggregates of orders $(15+3 k, 3)$ for any integer $k \geq 0$, the natural question is whether the $(18,3)$ aggregate of Example 4 can be constructed in this way. Interestingly, it cannot. The $(18,3)$ aggregate of Example 4 again dualizes to a set of points in the three planes $w=0, w=1$ and $w=\infty$, and even has the same finite points as in the $(15+3 k, 3)$ construction. However, the points in the plane at infinity (which we again project onto the $w=1$ plane) are strikingly 


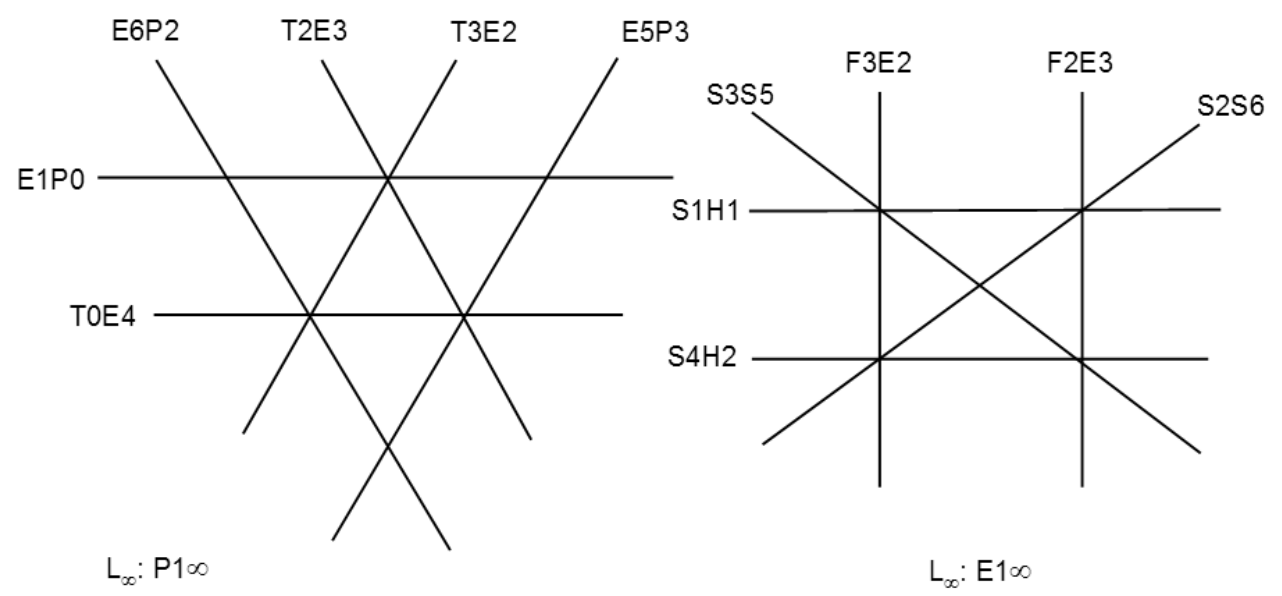

Figure 10: The detailed trace on a $T$-plane in Example 1 and the corresponding trace on $\Phi(T 1)$ in Example 2.

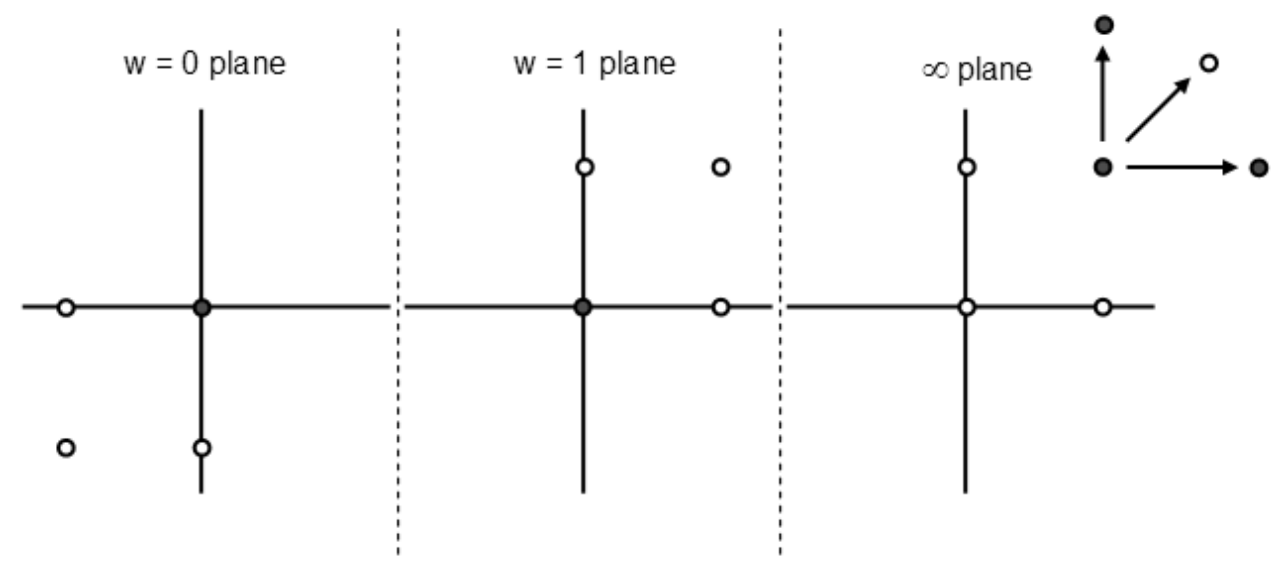

Figure 11: The point aggregate corresponding to Example 2 viewed as a series of planar layers. The points in the plane at infinity are projected onto the $w=1$ plane. The points in red are omittable. 
different (see Figure 13). Indeed, if the $(18,3)$ of Example 4 were obtainable from the previously described $(15+3 k, 3)$ construction then we would be able to remove three of the points to obtain a $(15,3)$, but it is readily verified that such a point deletion is not possible. Starting with the $(18,3)$ example we are able to go further and construct aggregates of orders $(19,3)$ and $(20,3)$ by adding points in the projected $w=\infty$ plane at $(1,0)$ and $(0,1)$ - in other words at the points $(1,0,1)_{\infty}$ and $(0,1,1)_{\infty}$. From there we can go even further to obtain aggregates of orders $(19+3 k, 3)$ and $(20+3 k, 3)$ for integer values of $k \geq 1$ by placing points in the projected $w=\infty$ plane at $\left(1+\frac{1}{k+1}, 1\right),\left(1,1+\frac{1}{k+1}\right),\left(1+\frac{1}{k+1}, 1+\frac{1}{k+1}\right)$. Hence we have the following:

Theorem 8 There exist sporadic aggregates of orders $(15,3)$ and $(18+k, 3)$ for any integer $k \geq 0$.

In order to push on to our next level of understanding of the sporadic aggregates let us consider the dual of the full $(39,7)$ aggregate of Example 3. See Figure 14. Recall that although we ultimately arrived at a $(33,7)$ aggregate, the construction began with a $(39,7)$ and we noticed that six of the planes (the $D$-planes) could be dropped without reducing the number of omittable planes. Unlike some of the previously considered aggregates, these aggregates seem like they would have been much harder to come up with purely from the point aggregate perspective. Here the $D$-planes correspond to the points in the $w=0$ plane together with the points $(1,1,0)_{\infty},(1,-1,0)_{\infty}$ in the plane at infinity (i.e. the only two points that project to infinite points in the $z=1$ plane). The $\langle 1,4,5\rangle$ pattern that we see in this example in the $w=-3, w=-2$ and $w=-1$ planes leads to a number of useful additional aggregates. For example, one can use the sequence $\langle 1,4,5,4,1\rangle$ to obtain a $(22,5)$ aggregate as shown in Figure 15. In actual fact, we found this aggregate while hunting for a small sporadic aggregate with exactly 4 omittable points. Only after constructing the aggregate did we realize that the point $(0,0,0)$ was omittable. However, after further consideration, we realized that the point $(0,0,0)$ can actually be deleted from the aggregate leaving an aggregate of order $(21,4)$, which is the smallest sporadic aggregate with exactly 4 omittable points that we know of. Note that, for example, the infinite point $(0,-1,1)_{\infty}$ remains omittable with respect to the point $(0,1,-1)$, even without $(0,0,0)$, by virtue of the point $(0,-1,1)$ - in other words, $\left\{(0,-1,1)_{\infty},(0,1,-1),(0,-1,1)\right\}$ are collinear. If we denote the resulting pattern in the finite planes by $\langle 1,4, \breve{4}, 4,1\rangle$, then with the same 7-point aggregate in the infinite plane (an aggregate which we henceforth denote by $\left.\Pi_{7}^{\infty}\right)$ we get additional aggregates with exactly 4 omittable points by considering finite planes with patterns $\langle 1,4, \breve{4}, 4, \breve{4}, 4,1\rangle,\langle 1,4, \breve{4}, 4, \breve{4}, 4, \breve{4}, 4,1\rangle$ and so on. In this way we obtain aggregates of orders $(21+8 k, 4)$ for any integer $k \geq 0$. We can obtain aggregates with exactly 5 omittable points by an analogous construction. We must simply insure that there is a central plane with the characteristic 5 point aggregate that, for example, appears in the $w=0$ plane in Figure 15 - a planar aggregate which we generically denote by $\Pi_{5}$. In addition to the four omittable points in the plane at infinity the central point of this central $\Pi_{5}$ will also be omittable. Thus, $\langle 1,4,5,4,1\rangle,\langle 1,4,5,4,5,4,5,4,1\rangle$ and $\langle 1,4,5,4,5,4,5,4,5,4,5,4,1\rangle$, each in combination with $\Pi_{7}^{\infty}$, are examples of aggregates of orders $(22+18 k, 5)$ for integer values of $k \geq 0$. 


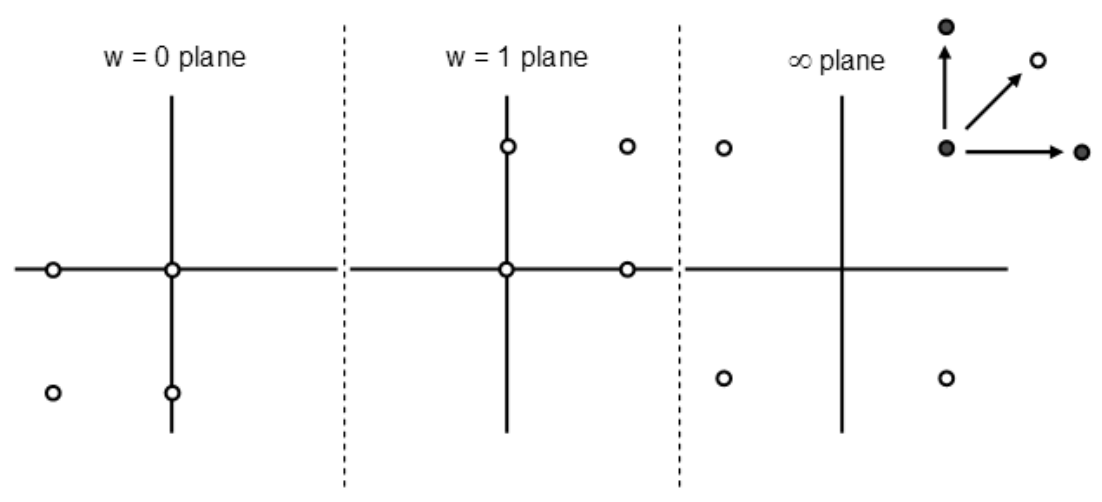

Figure 12: A $(15,3)$ point aggregate obtained by tinkering with the points in the plane at infinity so the finite omittable points in Figure 11 are no longer omittable. The remaining omittable points are again colored red.

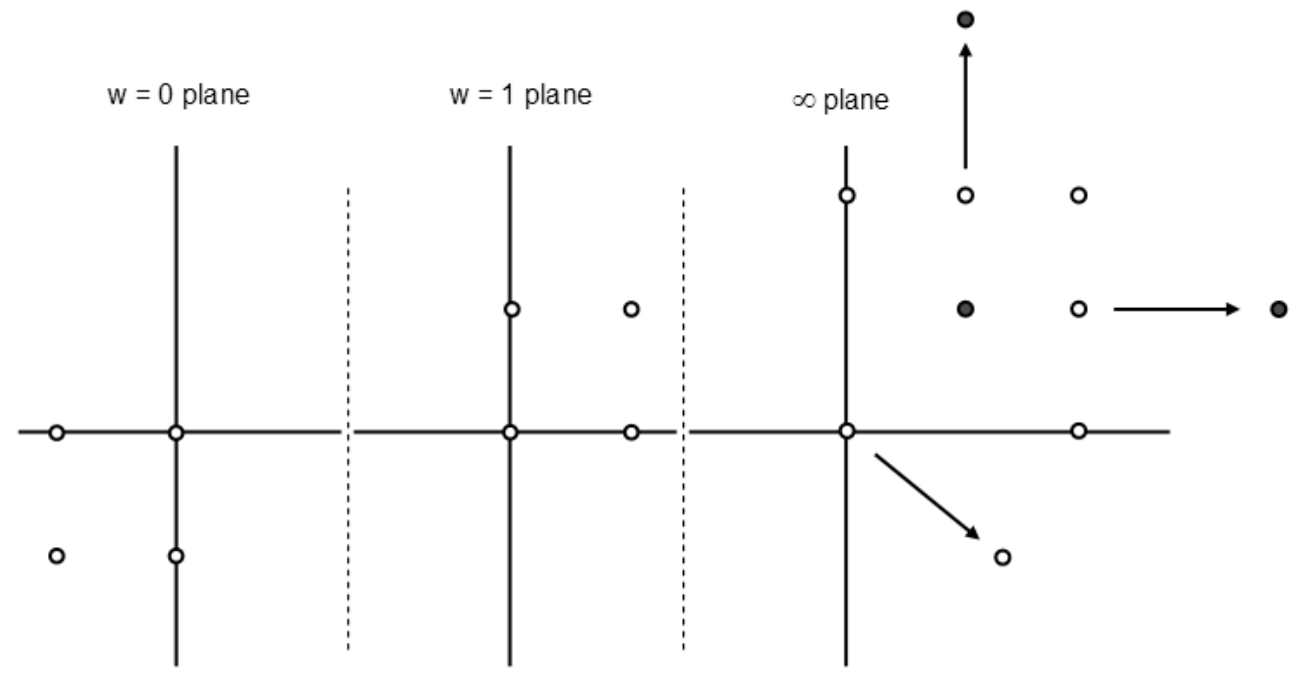

Figure 13: The $(18,3)$ point aggregate which is dual to the planar aggregate of Example 4. No three points of the aggregate may be removed keeping the designating omittable points omittable - proving that this aggregate is distinct from the aggregates constructed in the $(15+3 k, 3)$ construction. 

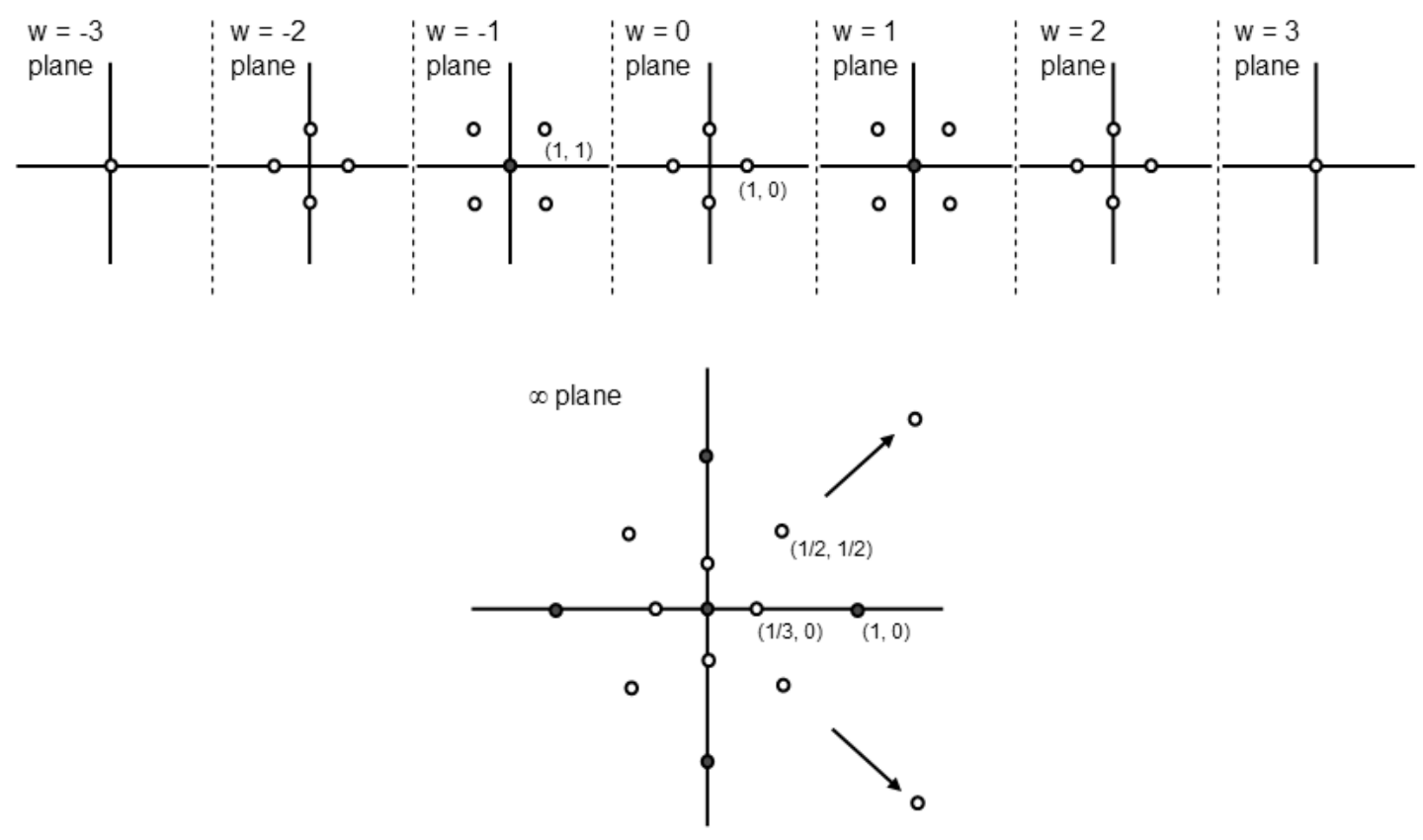

Figure 14: The $(39,7)$ point aggregate which is dual to the full (39-plane) planar aggregate of Example 3 under the duality transformation $\{(x, y, z): z=u x+v y-w\} \mapsto(u, v, w)$. The plane at infinity is presented projected onto the $w=1$ plane. The coordinates of several points are labeled for reference. The coordinates of other points can readily be inferred by symmetry.

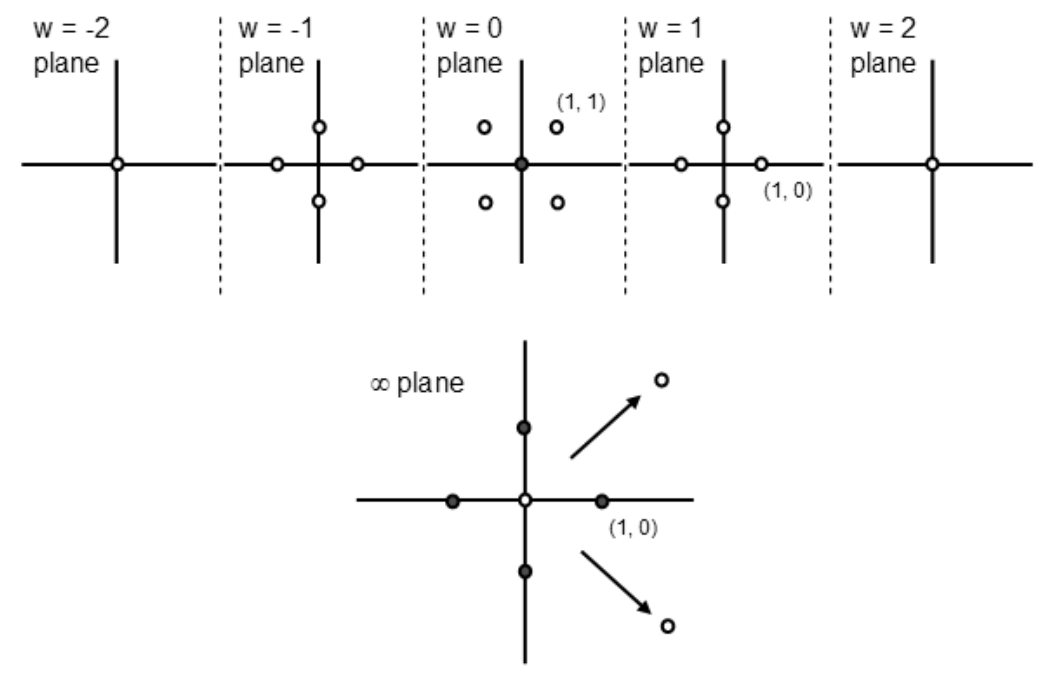

Figure 15: A $(22,5)$ aggregate which is an example of using the $\langle 1,4,5\rangle$ pattern (in this case a $\langle 1,4,5,4,1\rangle)$ revealed in the full $(39,7)$ example of Figure 14 . The plane at infinity is again presented projected onto the $w=1$ plane. As earlier, the coordinates of several points are labeled for reference. The coordinates of other points can be inferred by symmetry. The point $(0,0,0)$ can be deleted to obtain a $(21,4)$ aggregate. 
In fact there are actually many ways to mix and match these patterns. To get an aggregate with 5 omittable points it is just necessary that the central plane be a $\Pi_{5}$ - the other $\Pi_{5} \mathrm{~s}$ can be replaced with $\Pi_{\breve{4}} \mathrm{~s}$ - thus $\langle 1,4, \breve{4}, 4,5,4, \breve{4}, 4,1\rangle$ and $\langle 1,4, \breve{4}, 4, \breve{4}, 4,5,4, \breve{4}, 4, \breve{4}, 4,1\rangle$ also give rise to aggregates with 5 omittable points and slightly fewer total points - i.e. aggregates of orders $(22+16 k, 5)$. Alternatively, in the construction featuring the pattern $\langle 1,4,5,4,5, \ldots\rangle$ if there are an even number of $\Pi_{5} \mathrm{~s}$ then there is no central $\Pi_{5}$ plane and hence just the four omittable points at infinity. Examples are $\langle 1,4,5,4,5,4,1\rangle$ and $\langle 1,4,5,4,5,4,5,4,5,4,1\rangle$. We thus get aggregates of orders $(31+18 k, 4)$ for integer values of $k \geq 0$.

Let us summarize by saying simply that

Theorem 9 There exists a sporadic aggregate of order $(21,4)$ as well as sporadic aggregates with exactly 4 or 5 omittable points (planes) and an arbitrarily large number of total points (planes).

We wrap up our discussion by describing how to obtain sporadic aggregates with 6 or 7 omittable points (planes) and an arbitrarily large number of total points (planes). The construction of arbitrarily large sporadic aggregates with 6 omittable points is straight forward. Instead of using the plane at infinity we have been considering for the aggregates with 4 and 5 omittable points, we consider instead the plane at infinity from the $(39,7)$ aggregate in Figure 14. We can use this aggregate with or without the infinite points $(1,1,0)_{\infty}$ and $(1,-1,0)_{\infty}$, giving aggregates in the infinite plane with either 13 or 15 points - five of which will always be omittable (the five points shown in red). For future reference, let us refer to this plane at infinity without the points $(1,1,0)_{\infty},(1,-1,0)_{\infty}$ by $\Pi_{13}^{\infty}$. Let us first point out that pairing planes in the pattern $\langle 1,4,5,4,1\rangle$ with $\Pi_{13}^{\infty}$ does not leave the central point in the $\Pi_{5}$ plane omittable. If we call this central point $o=(0,0,0)$, then $o$ will be on ordinary lines with points like $\left(\frac{1}{2}, \frac{1}{2}, 1\right)_{\infty}$ and $\left(\frac{1}{3}, 0,1\right)_{\infty}$. However, if we consider a pattern of the form $\langle 1,4,5,4,5,4,5,4,1\rangle$ together with $\Pi_{13}^{\infty}$ the problem is rectified since the central point in the central $\Pi_{5}$ plane (a point which we shall continue to refer to as $o$ ) pairs with points in the left-most $\Pi_{5}$ plane to take care of directions like $\left(\frac{1}{2}, \frac{1}{2}, 1\right)_{\infty}$, while $o$ pairs with points in the left most $\Pi_{4}$ plane to take care of directions like $\left(\frac{1}{3}, 0,1\right)_{\infty}$. Analogously we can consider sequences of planes like $\langle 1,4,5,4,5,4,5,4,5,4,5,4,1\rangle$ or $\langle 1,4,5,4,5,4,5,4,5,4,5,4,5,4,5,1\rangle$ in conjunction with $\Pi_{13}^{\infty}$ to obtain aggregates of orders $(46+18 k, 6)$ for integer values of $k \geq 0$. Other than the central $\Pi_{5}$ we can replace any of the $\Pi_{5} \mathrm{~s}$ with $\Pi_{4} \mathrm{~s}$, and moreover, we can add either or both of the two infinite points $(1,1,0)_{\infty},(1,-1,0)_{\infty}$ to get additional examples. Also, by removing the central point at infinity, $(0,0,1)_{\infty}$ from the $(33,7)$ aggregate derived from Figure 14 (in other words, first removing the central $\Pi_{5}$ and the infinite points $(1,1,0)_{\infty},(1,-1,0)_{\infty}$ to obtain a $(33,7)$ and then removing $\left.(0,0,1)_{\infty}\right)$ we obtain a $(32,6)$ which is the smallest aggregate we have found with exactly 6 omittable points.

The construction showing that there are arbitrarily large sporadic aggregates with 7 omittable points is a bit more involved. In order to take the first step up from the example in Figure 14 in which we start with a $\langle 1,4,5,4,5,4,1\rangle$ we sandwich in an additional $\langle 4,5\rangle$ on the left, to the right of the leftmost $\Pi_{1}$, and sandwich an additional 
$\langle 5,4\rangle$ on the right, to the left of the rightmost $\Pi_{1}$. The central plane will still be a $\Pi_{4}$ but we will add points to the plane at infinity so that the central points in the adjacent $\Pi_{5} \mathrm{~s}$ remain omittable. The sequence of finite planes is $\langle 1,4,5,4,5,4,5,4,5,4,1\rangle$. If we consider the central point of the $\Pi_{5}$ immediately right of center, the only concerns about its connecting lines with other finite points are with respect to the points in the leftmost $\Pi_{4}$ and $\Pi_{5}$ - other connecting lines contain at least three points by symmetry considerations. To assure that the lines made by the center point of the $\Pi_{5}$ immediately right of center and the points on the leftmost $\Pi_{5}$ are not ordinary, we add points $\left( \pm \frac{1}{4}, \pm \frac{1}{4}, 1\right)_{\infty}$ and to assure that the lines made by the same center point and the points on the leftmost $\Pi_{4}$ are not ordinary, we add points $\left.\left(\frac{1}{5}, 0,1\right)_{\infty},\left(0, \frac{1}{5}, 1\right)_{\infty},-\frac{1}{5}, 0,1\right)_{\infty},\left(0,-\frac{1}{5}, 1\right)_{\infty}$. The result is illustrated in Figure 16. The collinearities needed to conclude that the infi-

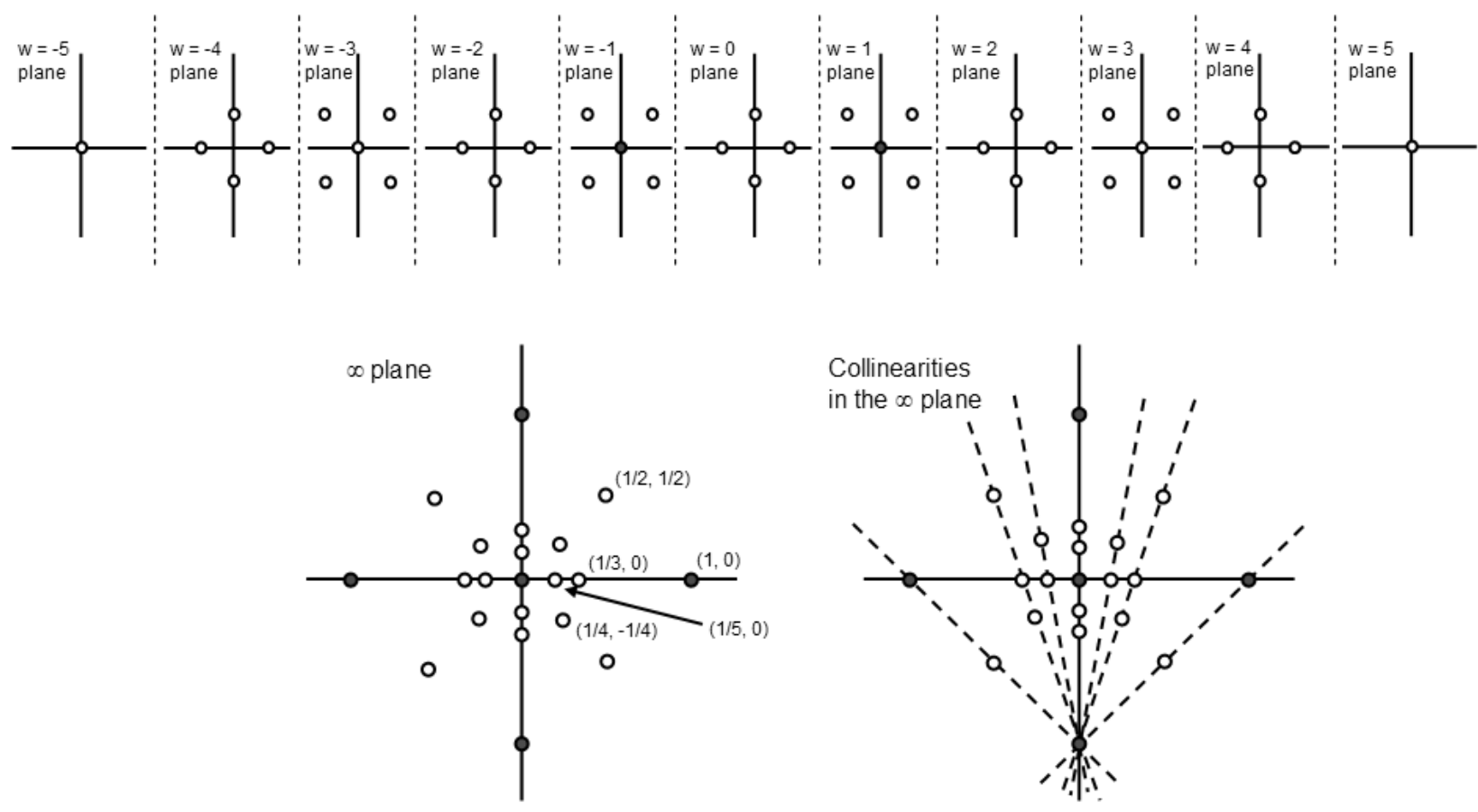

Figure 16: A $(63,7)$ aggregate obtained by extending the finite planes of Example 3, Figure 14 , to $\langle 1,4,5,4,5,4,5,4,5,4,1\rangle$ and then adding the infinite points $\left( \pm \frac{1}{4}, \pm \frac{1}{4}, 1\right)_{\infty}$ and $\left(\frac{1}{5}, 0,1\right)_{\infty},\left(0, \frac{1}{5}, 1\right)_{\infty},\left(-\frac{1}{5}, 0,1\right)_{\infty},\left(0,-\frac{1}{5}, 1\right)_{\infty}$. The infinite points $( \pm 1,1,0)_{\infty}$ have been dropped.

nite omittable points from Figure 14 remain omittable are shown in the bottom right hand drawing in the Figure. In order to obtain additional examples, at each iteration we sandwich in additional $\langle 4,5\rangle \mathrm{s}$ and $\langle 5,4\rangle_{\mathrm{s}}$ on the left and right in new parallel planes, and add points to the plane at infinity of the form $\left( \pm \frac{1}{2 k}, \pm \frac{1}{2 k}, 1\right)_{\infty}$ and $\left.\left(\frac{1}{2 k+1}, 0,1\right)_{\infty},\left(0, \frac{1}{2 k+1}, 1\right)_{\infty},-\frac{1}{2 k+1}, 0,1\right)_{\infty},\left(0,-\frac{1}{2 k+1}, 1\right)_{\infty}$. It is then sufficient to verify, in the plane, that the following points are collinear: $\left\{(0,1),\left(\frac{1}{2 k+1}, 0\right),\left(\frac{1}{2 k},-\frac{1}{2 k}\right)\right\}$ and $\left\{(0,1),\left(\frac{1}{2 k}, \frac{1}{2 k}\right),\left(\frac{1}{2 k-1}, 0\right)\right\}$, which serves, as in the case of the right-hand bottom diagram in Figure 16, to show that all five of the original omittable infinite points remain omittable 
with respect to points in the infinite plane in each successive new aggregate. We have thus established the following:

Theorem 10 There exists a sporadic aggregate of order (32,6) and arbitrarily large aggregates with either 6 or 7 omittable points (planes).

\section{$5 \quad$ Remarks and Open Problems}

1. It can readily be seen that the fundamental definition of omittability generalizes to all dimensions, as do several of the constructions from Section 2. A systematic treatment, including the identification of even a single sporadic example of omittable hyperplanes in the real projective space of dimension $k>3$ remains open.

2. Are there any infinite families of aggregates in addition to those articulated in Theorems 3 and 6 , for example where the omittable planes form a near-near-pencil (in other words, all planes but two pass through a common line)? Such an infinite family exists in $2 D$ - see [1], Theorem 6.

3. Are there additional sporadic aggregates with $g>7$ omittable planes?

4. The arrangements induced in the omittable planes constructed in Examples 1 - 4 by the traces of the other planes are simplicial. We do not know whether this phenomenon is accidental or if there is a hidden relationship.

5. An illustration of the difficulty of working with aggregates of planes in projective 3 -space is provided by the question of whether Examples 1 and 2, which we know to be isomorphic as aggregates, are projectively equivalent, or isomorphic as arrangements.

6. Going completely in the opposite direction from Theorem 5, can one obtain a lower bound on the number of planes relative to the number of omittable planes under the assumption that all omittable planes are in general position? The collections of omittable planes in Examples 1 and 4 are both in general position. While the omittable planes in Example 3 are not, if one deletes the plane at infinity to get a $(38,6)$ (or a $(32,6)$, if we first delete all $D$-planes), the six remaining omittable planes are in general position.

7. Are the aggregates we have found of orders $(21,4),(32,6)$ and $(33,7)$ actually minimal for the given number of omittable planes (i.e. for $g=4,6$ and 7 )? Can a sharpened theorem like Theorem 7 be found for $g>3(g \neq 5)$ ?

8. We are greatly indebted to Leah Berman for many comments, corrections and suggestions concerning earlier versions of this paper. 


\section{References}

[1] L. W. Berman, B. Grünbaum, and J. Lenchner. Omittable lines. Ars Math. Contemp., 2(1):206-222, 2008.

[2] J. Csima and E. Sawyer. There exist 6n/13 ordinary points. Discrete and Computational Geometry, 9:187-202, 1993.

[3] B. Grünbaum. Lectures on arrangements. University of Washington, Seattle (dittoed notes). Available at http://hdl.handle.net/1773/15699, 1974.

[4] B. Grünbaum and G. C. Shephard. Simplicial arrangements in projective 3-space. Mitt. Math. Semin. Giessen, 166:49-101, 1984.

[5] L. M. Pretorius and K. J. Swanepoel. The Sylvester-Gallai theorem, colourings and algebra. Discrete Mathematics, 309:385-399, 2009.

[6] J. E. Wetzel. Which Grübaum arragements are simplicial? Expositiones Math., 11:109-122, 1993. 